\title{
Research on Conservation Strategy of Traditional Villages Based on Information Technology
}

\author{
Liu Han ${ }^{1}$, Ai Yong ${ }^{2}$, Liu Xiaohu ${ }^{3}$ \\ ${ }^{1}$ Hubei Urban Construction Vocational and Technical College, Wuhan 430205, Hubei, China \\ ${ }^{2}$ Hubei Urban Construction Vocational and Technical College, Wuhan 430205, Hubei, China \\ ${ }^{3}$ School of Architecture and Urban Planning, Huazhong University of Science and Technology, Wuhan \\ 430074, Hubei, China \\ aliuhan66@163.com, ${ }^{\mathrm{b}}$ ai_echo@tom.com, ${ }^{\mathrm{c}} \operatorname{ltg} \mathrm{tgn} @$ sohu.com
}

\begin{abstract}
Keywords: New-type urbanization, Information technology, Traditional villages, Beautiful countryside, Conservation strategies.

Abstract. Under the background of new-type urbanization, the transformation of beautiful villages is a new requirement and a new topic for the new stage of urbanization in China. Using the research on traditional villages conservation strategy and information technology to establish traditional village database, the theories and methods that use information technology to conserve traditional villages are studied, which can promote the modernization process of planning management, can promote China's cultural heritage conservation synchronized with the world, and can adapt to the global trend of informtization and sustainable development.
\end{abstract}

\section{Introduction}

The conservation of smaller historic towns and traditional villages is an important part of cultural heritage conservation. Compared with urban cultural heritage conservation, smaller historic towns and traditional villages are rarely destroyed and have original ecology and relative integrity. In the world, attentions have always been paid to the conservation of smaller historic towns and traditional villages. The International Council on Monuments and sites (ICOMOS) respectively passed "Resolution on the Conservation of Smaller Historic Towns" in 1975 and "ICOMOS Declaration of Tlaxcala on the Revitalization of Small Settlements". which both proposed valuable suggestions on how to conserve and heritage historic towns and traditional villages.

There are a lot of historic towns and traditional villages in China because China has a long history with profound cultural background. They are not only the true portrayals of local traditional cultures, folk customs and architecture art that embodies the local cultures and spatial pattern of villages and towns, but also the reflections of venation of history, culture and social development, which is a matter of great significance for the inheritance of traditional culture and shaping the city cultural image and a valuable heritage of nonrenewable resources from our ancestors. However in recent years, many historic towns and villages ignored the scientific conservation of historic cultures during the process of economic development and large-scale construction. The traditional buildings are dilapidated and disrepair, and some areas dismantled old buildings to create new ones that are pseudo-classic architectures, besides, the phenomenon of "hollow villages" accelerated the decline and destroy of traditional villages, some new or renew houses built by villagers who are lack of awareness of conservation are inharmonious with traditional local styles, which destroyed the ancient customs and appearances, and even destroyed the traditional style and blocks construction of historical and cultural villages and towns to a treat extent. How to conserve historical and cultural towns and villages scientifically and effectively, how to handle the relationship between conservation and development, how to draw up guidelines for the conservation of historical and cultural towns and villages, and how to improve the cultural heritage planning and 
management and establish a long-term mechanism, has become a pressing matter of the moment.

\section{Application of GIS technology in traditional village conservation}

In the investigation of the revision of the traditional village conservation planning, the largest amount of work and data is the investigation of the existing buildings. In the investigation of the building, the basic properties of buildings such as times, layers, styles, structures, roof forms and construction quality are filed and building property right and use function are also stored in the database, besides, we stored the image data, such data is multidimensional and comprehensive. In a database, the building is numbered to help identify buildings and to correlate attribute data of the building to image data. If the number of existing buildings is larger, then the number of regional coding and building code can be achieved by the two stage numbering so that the latter management will be easier. According to the results of field investigation, a new layer was built in CAD topographic map and drew with corresponding codes around the building boundary, and then transferred to ArcGIS to form the present situation of building data layers, in its corresponding attribute table, the building number and building attribute values are automatic generated, the field storage of each building the number of completed status of architectural graphic data is input. Taking photos of each building and taking the building number as the directory name, and placing the photos in the appropriate directory for querying and generating the contents of the form. In the Excel table, enter the database status data of the building questionnaires, by linking the building number fields in the table with the building number fields in the current construction data layer (both data are identical), the architectural graphic data is associated with the attribute data; About the architectural image data association, through the query we can get the building numbers to get the specific location of the storage architecture of image data, and using graphics processing software to display the image query building shot so as to realize the architecture and image data association.

In the completed GIS database, you can query the building monomer information, including spatial information and basic attributes such as the numbers of layers, height, age, area, photos and so on. In addition, you can draw through the building monomer information table, and use the GIS software to export all the information of the building monomer. While from the connection into the building monomer information, according to different data layers, we can output all kinds of single factor information analysis chart as a conservation plan of the building status analysis diagram. Compared with the traditional CAD drawing method, the information technology has obvious advantages in modifying and quantizing. It can be changed from the data source, and also can reflect the status information of the building in the GIS. The same method can also be used in the establishment of all streets and lanes database.

\section{The combination of ArcGIS and SketchUp in the creation of information model}

Compared with two bit graphics and data, browsing data in 3D scene is more intuitive and real, which can provide some information that not be obtained directly on the plane. The terrain and structures of the area can also be visually visualized. The ArcGIS 3D analysis extension provides tools for 3D visualization, analysis, and surface production. The ArcScene application is the core extension module of ArcGIS 3D analysis. Using three-dimensional analysis, we can manage 3D, GIS data, process 3D analysis, edit 3D elements, create 3D layers, and generate two-dimensional data into 3D elements and other functions.

SketchUp building sketch design tools are widely used and easy to operate. It gives architects the experience of edge design and performance, rapidly forms architectural sketches, and creates architectural schemes. Similar to the process of drawing sketches manually by designers, the finished product can import other coloring, posting and rendering software at the same time and continue to form 
a photo based business effect diagram.

In the combination of ArcScene (ArcGIS) and SketchUp information, the SketchUp can convert 2D GIS data into 3D data, and the SketchUp can quickly build real buildings, building components, blocks, equipments, people, terrains and other various types of 3D model. Moreover, more importantly, the seamless integration of such SketchUp with ArcGIS enables the stereoscopic model to contain attribute information.

The combination of ArcScene and SketchUp has the following advantages:

(1) The operations of SketchUp and ArcScene are relatively simple and very suitable for construction engineers.

(2) The compatibility of SketchUp and ArcScene is good, and you can support the SketchUp model with maps to import ArcGIS to make the effects more realistic.

(3) The group model established in SketchUp can not only modify and assign the attribute fields after importing ArcGIS, but also establish attribute fields and assign values in SketchUp.

There are also some problems associated with the combination of ArcScene and SketchUp. For example, it is unable to realize the dimension drive function, which didn't form a dynamic link between value in the dimension field and the actual numeral field; It is not as perfect BIM system of this kind of Revit with perfect structural system, and it has the lack of standardization of modeling.

\section{Creation of information model based on Revit}

The REVIT of information modeling has a qualitative leap from the traditional concept of 3D modeling, and its greatest advantage is the combination of spatial information with non-spatial information using the idea of GIS (geographic information system). But the traditional 3D modeling only establish a three-dimensional model in the computer, if there is no subjective judgment, computer will not tell us names, materials, time and other non-spatial information of each graphical model. With the idea of GIS, BIM will tell us the size, horizontal position, vertical position and other spatial information and materials, its name, and other non-spatial information (attributes) when we are clicking on a certain building model.

The application of information modeling in the conservation of traditional villages are still at the initial stage without an unified standard; in the conservation of traditional villages, we try to make some BIM of the ancient buildings in the project of traditional villages conservation informatization, there are some combinations of graphic and attribute values. The clans of building components created in REVIT can be sized to drive.

The advantage of using REVIT to build an information-based model is obvious:

(1) REVIT has a good parametric modeling technique -- size driven, enabling dynamic transformations of graphics and sizes.

(2) REVIT has a more mature platform to ensure the quality of the model.

(3) The clan concept of REVIT can make modeling like standard structural programming; building a model then next time if we need a model for the same type but different size, what we should do is only to modify the size, REVIT will use the dimension driven to automatically generate the models, greatly improve the efficiency.

(4) REVIT can establish three-dimensional information model through two-dimensional drawing, and automatically generate planar graph, vertical graph and sectional graph to meet the needs of the project.

(5) The size of ancient buildings is very strict in general with strict modulus, which is more suitable for parametric modeling of REVIT.

Of course, the application of REVIT in the traditional village conservation is still in its infancy, and there are some problems in the process of combining with ArcGIS.

Generally, the application of REVIT in the traditional villages conservation is still in its infancy, and 
there are some problems in the process of combining with ArcGIS. First, compared with the SketchUp software, REVIT learning is very difficult; although with the rapid understanding, we can draw simple graphics, but if you want to establish the model of ancient building information and ensure the completeness and accuracy of the information, it will be not just a simple draw can achieve, and it must have profound knowledges and rich REVIT practical engineering exercise, which can realize the building information model rather than simple three-dimensional model. In addition, the REVIT interface with ArcGIS has not made any headway. Because the concept of BIM is a new concept (of course, for GIS, it can only be said to be an extension), and there are few examples of integration with GIS, therefore the interface becomes a big problem at the moment.

\section{Conclusion}

In the process of study on the conservation strategies of traditional villages, working with enterprises and using a variety of social resources in enterprises, we do experimental productions for suggested building environment; after the actual operation, we can find the lack of theoretical researches and software models and revise and optimize the results, so that it can be seamlessly in docking with the actual projects the future. In cooperation with local governments, through close exchanges and cooperation with the local government with experiences of real management and implementation of rural planning and construction, we can understand the latest policies, the new specific problems and the need of coordination of the contradiction, which can make this research more targeted and practical, make strategies and schemes more feasible to improve the practical significance of the study.

Using information technology to build traditional village database. The powerful spatial analysis function of database can not only complete data input, storage and management of historic cultural heritage surveys, use current database to draw up status analysis chart of conservation areas, and use attribute database associated with graphics, we can achieve two-way query, retrieval and analysis of graphics and attribute data; besides, the spatial analysis function of database can also conduct comprehensive evaluation and judgement based on multi-source data integration, so that we can avoid faults in decision making of conservation planning because of cognitive limitation and provide decision makers with multiple conservation methods in order to improve the scientific and comprehensive nature of conservation planning structure. At the same time, through information technology, it can be used as a means of communicating conserving strategies with the public and promoting cooperation with other government departments within or near the heritage resources to broaden the levels of public participation.

\section{References}

[1] Wang Yansong, Lv Xiaohang. Ecological suitability analysis of site selection and layout of traditional mountain villages based on GIS. Central China architecture. 2011.10

[2] Wang Chao. Application of information technology in the protection of ancient villages. Dissertation database.2007 of Xi'an University Of Architecture And Technology

[3] Dang an Rong. Information technology method of traditional village protection. China Construction Information.2013.11

[4] Jian Ning Zhu Xia. Further understanding of traditional village culture planning new normal under the background of.2015 China city planning conference proceedings.2015

[5] Dong Wei, the past and present of a traditional village -- the relevance of new technology and conservation concept to the conservation planning of the village in Yueqing, South China. Architect.2005.3 